\title{
A CONVENIENT SYNTHESIS OF PYRROLO|3,4-c|QUINOLINES
}

\author{
Miklós Nyerges*, Andrea Virányi and László Tơke \\ Research Group of the Hungarian Academy of Sciences, Department of Organic Chemical Technology, Technical \\ University of Budapest, H-1521 Budapest P.O.B. 91, Hungary
}

\begin{abstract}
A new route to the pyrrelof $3,4-c$ ]quinoline ring system has been developed. The synthesis proceeds stereoselectively in three steps, using 1,3-dipolar cycloaddition of azomethine ylides as a key step. First, a series of 4-aryl-pyrrolidine-3carboxylic acid has been prepared from the appropriate cinnamic esters and a non-stabilised azomethine ylide. The reduction of nitro group on the aromatic ring was followed by the acid catalyzed intramolecular lactame formation.
\end{abstract}

\section{Introduction}

Pyrrolo[3,2-c]quinoline derivatives represents the central core a number of biologically significant molecules: for example, 7-chloro-3-methyl-1 $H$-pyrrolo[3,2-c]quinoline-4-carboxylic acid 1 was found to be a relatively potent and selective inhibitor of kynurenine-3-hydroxylase (1), or certain compounds belonging to this class display antineoplastic activities(2). Martinellic acid 2 and martinelline 3, isolated from the roots of the tropical plant Martinella iquitosensis, are the first alkaloids with the pyrrolo[3,2-c]quinoline ring system displaying unique biological activity, as they are the first naturally occurring nonpeptide bradykinin $B_{1}$ and $B_{2}$ receptor antagonists (3). A number of syntheses of these heterocycles have been reported (4), which also includes our own work (5). However, there have been only a few reports on the synthesis of the closely related pyrrolo[3,4-c]quinolines and in addition none of them generally applicable to the synthesis of these heterocycles (6). Here we report the results of our efforts in this direction.<smiles>Cc1c[nH]c2c1cnc1cc(Cl)ccc12</smiles>

1<smiles>[R][R]#[R][R]</smiles>

Scheme 1

\section{Results and Discussion}

This approach, which is illustrated in Scheme 2 in a retrosynthetic manner, involves the disconnection of the lactam bond of 4 followed by a functional group interconversion $\left(\mathrm{NH}_{2} \Rightarrow \mathrm{NO}_{2}\right)$ to afford the 3-(2-nitro-aryl)-pyrrolidine 5. An intermolecular [3+2] azomethine ylide-alkene cycloaddition would produce the wanted pyrrolidine 
<smiles>[X]c1cccc(C2C(C(=O)N(C)C)C([R])N([R])C2[R])c1</smiles><smiles>[R]OC(=O)C1C(c2ccc([X])cc2[N+](=O)[O-])C([R])N([R])C1[R]</smiles><smiles>[R]C=[N+]([R])[B-]([R])=CC</smiles>

Scheme 2

Among the vast amount of available azomethine ylides we choose to investigate the decarboxylative condensation of sarcosine with paraformaldehyde which results in the formation of one of the most simple dipole of this type (7). As it appeared from the literature only Joucla and Mortier studied this kind of azomethine ylide addition to methyl cinnamate (8), beside a few similar cycloadditions of other more complicated azomethine ylides to this olefine generated by different methods (9).

A solution of the dipolarophile and an excess of sarcosine and paraformaldehyde were heated under reflux in the appropriate solvent (the water formed was removed by the aid of a Dean-Stark trap) which resulted in formation of the corresponding cycloadducts (Scheme 3, Table 1) after 1.5 - 5 hours in moderate to good yields. We choose to investigate a few additional examples (Entries 3-5) to demonstrate the effect of different kinds of substituents beared by the aromatic ring on the reactivity of dipolarophiles.

It can be concluded from the data of Table 1, that the ethyl cinnamates having electron donating substituents on the aromatic ring (Entries 5-7) are less reactive than those without, as they have reacted only in boiling xylene. This is in good agreement with our earlier results (7c).

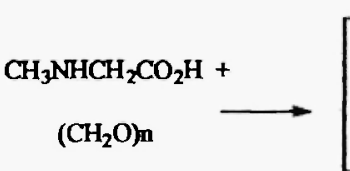<smiles>C=[N+](C)[O-]</smiles>

8

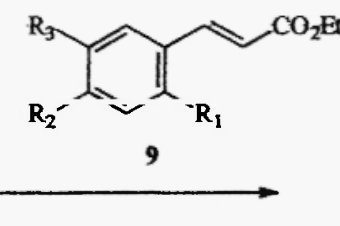

Scheme 3<smiles>[R]COC(=O)C1CN(C)CC1c1cc([R])c([R])cc1[R]</smiles>

10

\begin{tabular}{|c|c|c|c|c|c|c|c|c|}
\hline Entry & $\overline{R_{I}}$ & $R_{2}$ & $\boldsymbol{R}_{\mathbf{3}}$ & Sotvent & $T$ & $\begin{array}{c}\text { Reaction } \\
\text { time }\end{array}$ & Product & Yield \\
\hline $1 *$ & $\mathbf{H}$ & $\mathbf{H}$ & $\bar{H}$ & Benzene & $80 C^{\circ}$ & $3 \mathrm{~h}$ & $10 a$ & $58 \%$ \\
\hline 2 & $\mathrm{NO}_{2}$ & $\mathbf{H}$ & $\mathbf{H}$ & Toluene & $110 \mathrm{C}^{\circ}$ & $1.5 \mathrm{~h}$ & $10 \mathrm{~b}$ & $85 \%$ \\
\hline 3 & $\mathbf{H}$ & $\mathbf{H}$ & $\mathrm{NO}_{2}$ & Tolucne & $110 \mathrm{C}^{\circ}$ & $1.5 \mathrm{~h}$ & $10 c$ & $79 \%$ \\
\hline 4 & $\mathbf{H}$ & $\mathrm{Cl}$ & $\mathbf{H}^{2}$ & Toluene & $110 C^{\circ}$ & $2 \mathrm{~h}$ & lod & $33 \%$ \\
\hline 5 & $\mathbf{H}$ & OMe & OMe & Xylene & $141 \mathrm{C}^{\circ}$ & $3 \mathrm{~h}$ & $10 \mathrm{e}$ & $44 \%$ \\
\hline 6 & $\mathrm{NO}_{2}$ & OMe & OMe & Xylene & $141 \mathrm{C}^{\circ}$ & $5 \mathrm{~h}$ & $10 f$ & $74 \%$ \\
\hline 7 & $\mathrm{NO}_{2}$ & \multicolumn{2}{|c|}{$\mathrm{OCH}_{2} \mathrm{O}$} & Xylene & $141 C^{\circ}$ & $3 \mathrm{~h}$ & $10 \mathrm{~g}$ & $56 \%$ \\
\hline
\end{tabular}

* experiment of Joucla and Mortier?

Table 1. Synthesis of pyrrolidin-carboxylic acids 10 via Scheme 2 
The appropriately substituted cycloadducts $10 \mathrm{~b}, 10 \mathrm{f}, 10 \mathrm{~g}$ were hydrogenated in the presence of $\mathrm{Pd} / \mathrm{C}$ catalyst to yield the corresponding anilines $11 \mathrm{a}-\mathrm{c}$. These intermediates were further cyclized in the presence of $p$-toluenesulphonic acid in boiling ethanol to give the targeted heterocycles 12a-c (10).

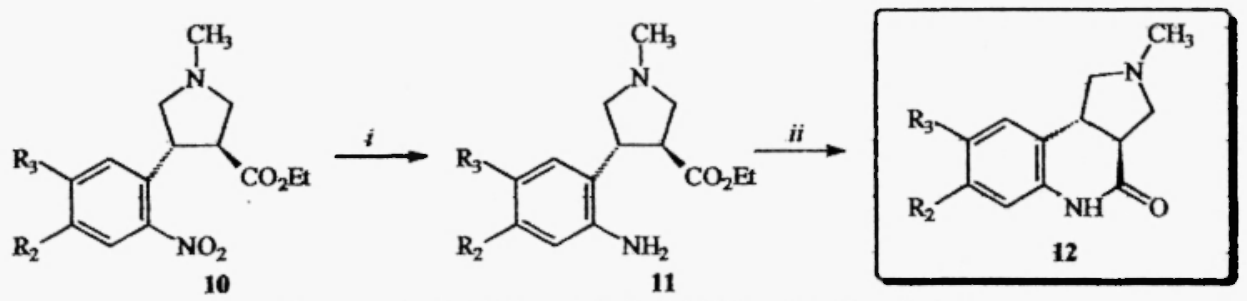

Scheme $4 i$.Pd/C, $\mathrm{H}_{2}$, EtOH; ii.PTSA, EtOH, reflux;

\begin{tabular}{c|ccccc}
\hline Entry & $\begin{array}{c}\text { Starting } \\
\text { cycloadduct }\end{array}$ & Product & $\boldsymbol{R}_{2}$ & $\boldsymbol{R}_{3}$ & Yield \\
\hline 1 & $10 \mathrm{~b}$ & $12 \mathrm{a}$ & $\mathrm{H}$ & $\mathrm{H}$ & $90 \%$ \\
2 & $10 \mathrm{f}$ & $12 \mathrm{~b}$ & $\mathrm{OMe}$ & $\mathrm{OMe}$ & $92 \%$ \\
3 & $10 \mathrm{~g}$ & $12 \mathrm{c}$ & $\mathrm{OCH}_{2} \mathrm{O}$ & $87 \%$ \\
\hline
\end{tabular}

Table 2 Synthesis of pyrrolo[3,4-c]quinolin-4-ones (12);

In conclusion, we have developed a new, three-step, stereoselective route from simple starting materials to hardly accessible pyrrolo[3,4-c]quinoline ring system. Work is currently being undertaken in our laboratory to further explore the scope and limitation of this route for the construction of pyrrolo[3,4-c]quinoline involving of more complicated azomethine ylides and other cinnamic acid derivatives.

Acknowledgments- This work was financially supported by the National Found for Science and Research, Hungary (OTKA Project No. T 032221). N.M. thanks the Ministry of Education for a Gybrgy Békésy fellowship.

\section{References}

(1) Heidempergher, F.; Pevarello, P.; Pillan, A.; Pinciroli, V.; Della Torre, A.; Speciale, C. Marconi, M.; Cini, M.; Toma, S.; Greco, F.; Varasi, M.; Il Farmaco 54, 1523 (1999).

(2) Badawey E.S.A.M.; Kappe, T.; Eur. J. Med. Chem. 32, 815 (1997).

(3) Witherup, K.; Ranson, R.W.; Graham, A.C.; Barnard, A.M.; Salvatore, M.J.; Limma, W.C.; Anderson, P.S.; Pitzenberger, S.M.; Varga, S.L.; J. Am. Chem. Soc. 117, 6682 (1995).

(4) (a) He, Y.; Mahmud, H; Wayland, B.R; Dias, H.V.R; Lovely C.J.; Tetrahedron Letters 43, 1171 (2002). (b) Ruano, J.L.G.; Tito, A.; Peromingo, M.T.; J. Org. Chem. 67, 981 (2002). (c) Snider, B.B.; Ahn, Y.; O'Hare, S.M.; Org. Lett. 3, 4217 (2001). (d) Batey, R.A.; Powell, D.A.; J. Chem. Soc. Chem. Commun. 2362 (2001). (e) Browning, R.G.; Mahmud, H.; Badarinarayana, V.; Lovely, C.J.; Tetrahedron Letters 42, 7155 (2001). (f) Hadden, M.; Nieuwenhuyzen, M.; Osborne, D.; Stevenson, P.J.;Thompson, N.; Tetrahedron Letters 42, 6417 (2001). (g) Ma, D.; Xia, C.; Jiang, J.; Zhang, J.; Org. Lett. 3, 2189 (2001). (h) Hadden, M.; Nieuwenhuyzen, M.; Potts, D.; Stevenson, P.J.;Thompson, N.; Tetrahedron 57, 5615 (2001). (i) Mahmud, H.; Lovely C.J.; Dias, H.V.R: 
Tetrahedron 57, 4095, (2001). (j) Snider, B.B.; O'Hare S.M.; Tetrahedron Letters 42, 2455 (2001). and references cited therein.

(5) Fejes I.; Nyerges, M.; Tõke, L.; Tetrahedron Lett. 40, 7951 (2000).

(6) (a) Horner, L.; Justus Liebigs Ann. Chem. 548, 117, (1941). (b) Roy, R. B.; Swan, G. A.; Chem. Commun. 1446 (1968). (c) Roy, R B.; Swan, G. A.; J. Chem. Soc. C, 1886 (1969). (d) Murata, G.; Satoru, H.; Teramato, K.; Miura, M.; Nomura, M.; Heterocycles, 36, 2147 (1993). (e) Swan, G. A.; Roy, R.B.; Khandelwal, G. D., J. Chem. Soc. 891 (1974). (f) Jones, K.; Wilkinson, J.; J. Chem. Soc. Chem. Commun, 24, 1767 (1992). (g) Veenstra, S. J.; Fortgens, R.; Vijn, R. J.; Jong, B. S. ; Speckamp, W. N.; Tetrahedron, 43, 1147 (1987). (h) Gündel, W.-F.; Bohnert, S.; Z. Naturforsch. 40b, 1409 (1985).

(7) (a) Tsuge, O.; Kanemasa, S.; Ohe, M.; Takenaka, S. Chem.Lett. 973 (1986). (b) Tsuge, O.; Kanemasa, S.; Ohe, M.; Takenaka, S. Bull. Chem. Soc. Jpn. 60, 4079 (1987). (c) Nyerges, M.; Balázs, L.; Bitter, 1.; Kádas, I.; Kövesdi, I.; Tổke, L. Tetrahedron 51, 6783 (1995).

(8) Joucla, M.; Mortier, J.; Bull. Soc. Chim. Fr. 579 (1988).

(9) (a) Padwa, A.; Chen, Y.-Y.; Dent, W.; Nimmesgern; H.; J. Org. Chem. 50, 4006 (1985). (b) Fevig, J.M.; Abelman, M.M.; Britelli, D.L.; Kettner, C.A.; Knabb, R.M.; Weber, P.C.; Bioorg. Med. Chem. Lett. 6, 295 (1996). (c) Fevig, J.M.; Buriak, J.; Stouten, P.F.W.; Knabb, R.M.; Lam, G.N.; Wong, P.C.; Wexler, R.R; Bioorg. Med. Chem. Lett. 9, 1195 (1999). (d) Hosomi, A.; Sakata, Y.; Sakurai, H; Chem. Lett. 1117 (1984). (e) Dubuffet, T.; NewmanTancerdi, A.; Cussac, D.; Audinot, V.; Loutz, A.; Millan, M.J.; Lavielle, G.; Bioorg. Med. Chem. Lett. 9, 2059 (1999).

(10) Selected spectroscopical data for representative compounds: Ethyl, N-Methyl-4-(2-nitrophenyl)pyrrolidine-3carboxylate (10b); 'H-NMR (250 MHz, $\mathrm{CDCl}_{3}$ ): 7.80 (d, $\left.1 \mathrm{H}, J 7.9 \mathrm{~Hz}, \mathrm{Ar}-3{ }^{\prime} \mathrm{H}\right), 7.72$ (d, $1 \mathrm{H}, J 7.9 \mathrm{~Hz}, \mathrm{Ar}-6$ ' $\mathrm{H}$ ), $7.58(\mathrm{t}, 1 \mathrm{H}, J 7.9 \mathrm{~Hz}, \mathrm{Ar}-5$ ' $\mathrm{H}), 7.34\left(\mathrm{t}, 1 \mathrm{H}, J 7.9 \mathrm{~Hz}, \mathrm{Ar}-4{ }^{\prime} \mathrm{H}\right), 4.12\left(\mathrm{~m}, 3 \mathrm{H}, \mathrm{OCH}_{2}\right.$ and $\left.\mathrm{H}-4\right), 3.23(\mathrm{t}, 1 \mathrm{H}, J 8.2 \mathrm{~Hz}$, H-2), $3.15-2.84(\mathrm{~m}, 3 \mathrm{H}, \mathrm{H}-2, \mathrm{H}-3, \mathrm{H}-5), 2.73(\mathrm{t}, 1 \mathrm{H}, J 7.9 \mathrm{~Hz}, \mathrm{H}-5), 2.42\left(\mathrm{~s}, 3 \mathrm{H}, \mathrm{NCH}_{3}\right), 1.21(\mathrm{t}, 3 \mathrm{H}, J 7.2 \mathrm{~Hz}$, $\mathrm{CH}_{2} \mathrm{CH}_{3}$ ); ${ }^{13} \mathrm{C}-\mathrm{NMR}\left(63 \mathrm{MHz}, \mathrm{CDCl}_{3}\right.$ ): 173.1 (q, C=O), 149.7 (q, $\left.\mathrm{Ar}-2^{\prime} \mathrm{C}\right), 139.0$ (q, Ar-l' C), $132.9\left(\mathrm{CH}, \mathrm{Ar}-5^{\prime} \mathrm{C}\right.$ ), 129.1 (CH, Ar-6’C), 127.1 (CH, Ar-4’ $\left.{ }^{\prime}\right), 123.6\left(\mathrm{CH}, \mathrm{Ar}-3^{\prime} \mathrm{C}\right), 63.8\left(\mathrm{CH}_{2}, \mathrm{C}-5\right), 60.9\left(\mathrm{OCH}_{2}\right), 59.5\left(\mathrm{CH}_{2}, \mathrm{C}-3\right)$, 52.6 (CH, C-4), $41.7\left(\mathrm{NCH}_{3}\right), 41.6(\mathrm{CH}, \mathrm{C}-3), 13.9\left(\mathrm{CH}_{2} \mathrm{CH}_{3}\right) ; \mathrm{R}\left(\mathrm{KBr}, \mathrm{cm}^{1}\right): 2970,2795,1729,1527,1379$, 1358, 1342, 1319, 1242, 1174, 1029; Ethyl, N-Methyl-4-(2-aminophenyl)pyrrolidine-3-carboxylate (11a); ${ }^{\mathrm{H}-\mathrm{NMR}}$ $\left(250 \mathrm{MHz}, \mathrm{CDCl}_{3}\right.$ ): 7.06 (t, 1H, J $\left.6.8 \mathrm{~Hz}, \mathrm{Ar}-5^{\prime} \mathrm{H}\right), 7.01$ (t, IH, $\left.J 6.8 \mathrm{~Hz}, \mathrm{Ar}-4{ }^{\prime} \mathrm{H}\right), 6.64$ (d, 1H, J 6.8 Hz, Ar-6'H), $6.58\left(\mathrm{~d}_{3} 1 \mathrm{H}, J 6.8 \mathrm{~Hz}, \mathrm{Ar}-3^{\prime} \mathrm{H}\right), 4.99$ (br s, $\left.2 \mathrm{H}, \mathrm{NH}_{2}\right), 4.13$ (q, $2 \mathrm{H}, J 7.1 \mathrm{~Hz}, \mathrm{OCH}_{2}$ ), 3.71 (m, 1H, H-4), $3.35-3.12$ (m, 2H, H-2, H-5), 3.04 (dd, $1 \mathrm{H}, J 3.0$ and $9.6 \mathrm{~Hz}, \mathrm{H}-2), 2.82-2.52(\mathrm{~m}, 2 \mathrm{H}, \mathrm{H}-3, \mathrm{H}-5), 2.38\left(\mathrm{~s}, 3 \mathrm{H}, \mathrm{NCH}_{3}\right), 1.22(\mathrm{t}$, $3 \mathrm{H}, J 7.2 \mathrm{~Hz}, \mathrm{CH}_{2} \mathrm{CH}_{3}$ ); ${ }^{13} \mathrm{C}-\mathrm{NMR}\left(63 \mathrm{MHz}, \mathrm{CDCl}_{3}\right.$ ): 174.1 (q, C=0), 144.6 (q, $\mathrm{Ar}-2{ }^{\prime} \mathrm{C}$ ), 129.5 (CH, Ar-6'C), 127.6 (q, Ar-1'C), $127.2\left(\mathrm{CH}, \mathrm{Ar}-4^{\prime} \mathrm{C}\right), 117.0\left(\mathrm{CH}, \mathrm{Ar}-5^{\prime} \mathrm{C}\right), 115.7\left(\mathrm{CH}, \mathrm{Ar}-3^{\prime} \mathrm{C}\right), 60.9\left(\mathrm{CH}_{2}, \mathrm{C}-5\right), 60.5\left(\mathrm{OCH}_{2}\right)$, $59.7\left(\mathrm{CH}_{2}, \mathrm{C}-3\right), 49.2(\mathrm{CH}, \mathrm{C}-4), 44.4(\mathrm{CH}, \mathrm{C}-3), 41.0\left(\mathrm{NCH}_{3}\right), 13.9\left(\mathrm{CH}_{2} \mathrm{CH}_{3}\right)$; IR (neat, $\left.\mathrm{cm}{ }^{1}\right): 3441,3185,2976$, 2788, 1728, 1644, 1497, 1457, 1247, 1184, 1158, 1033; 2-Methyl-1,2,3,3a,5,9b-hexahydro-pyrrolo[3,4-c]quinolin4-one (12a); ${ }^{1} \mathrm{H}-\mathrm{NMR}\left(250 \mathrm{MHz}, \mathrm{CDCl}_{3}\right.$ ): 9.10 (br s, $\left.1 \mathrm{H}, \mathrm{NH}\right), 7.20$ (m, 2H, Ar-H), 7.01 (d, $1 \mathrm{H}, J 7.8 \mathrm{~Hz}, \mathrm{Ar}-\mathrm{H}$ ), $6.89(\mathrm{~d}, \mathrm{IH}, J 7.8 \mathrm{~Hz}, \mathrm{Ar}-\mathrm{H}), 3.35(\mathrm{~m}, 2 \mathrm{H}, \mathrm{H}-3 a$ and $\mathrm{H}-9 b), 3.20(\mathrm{~d}, 2 \mathrm{H}, J 9.9 \mathrm{~Hz}, \mathrm{H}-3), 3.03(\mathrm{~d}, 2 \mathrm{H}, J 9.7 \mathrm{~Hz}, \mathrm{H}-$ 1), 2.57 (s, 3H, $\mathrm{NCH}_{3}$ ); ${ }^{13} \mathrm{C}-\mathrm{NMR}\left(63 \mathrm{MHz}, \mathrm{CDCl}_{3}\right.$ ): 171.9 (q, C=O), 138.1 (q, C-5a), 122.4 ( $\left.\mathrm{CH}, \mathrm{C}-7\right), 127.0$ (q, $\mathrm{C}-9 a), 124.4(\mathrm{CH}, \mathrm{C}-9), 123.0(\mathrm{CH}, \mathrm{C}-8), 115.7(\mathrm{CH}, \mathrm{C}-6), 56.4\left(\mathrm{CH}_{2}, \mathrm{C}-1\right), 52.8\left(\mathrm{CH}_{2}, \mathrm{C}-3\right), 46.4(\mathrm{CH}, \mathrm{C}-3 a)$, $43.6\left(\mathrm{NCH}_{3}\right), 42.1(\mathrm{CH}, \mathrm{C}-96)$; IR (neat, $\left.\mathrm{cm}^{-1}\right): 3436,3206,2766,1678,1480,1401,1274,1248,1186,1112$;

\section{Received on January 17, 2003}

